\title{
A Note on a Combinatorial Conjecture
}

\author{
Guixin Deng \\ School of Mathematical Science, Guangxi Teachers Education University, Nanning, China \\ Email: dengguixin@live.com
}

Received October 7, 2012; revised November 7, 2012; accepted November 17, 2012

\begin{abstract}
It is difficult to find Boolean functions achieving many good cryptographic properties. Recently, Tu and Deng obtained two classes of Boolean functions with good properties based on a combinatorial conjecture about binary strings. In this paper, using different approaches, we prove this conjecture is true in some cases. This conjecture has resisted different attempts of proof since it is hard to find a recursive method. In this paper we give a recursive formula in a special case.
\end{abstract}

Keywords: Binary String; Weight

\section{Introduction}

Let $x$ be a nonnegative integer. If the binary expansion of $x$ is

$$
x=\sum_{i} x_{i} 2^{i}
$$

then the Hamming weight of $x$ is

$$
w=\sum_{i} x_{i}
$$

In [1] Tu and Deng proposed the following conjecture.

Conjecture 1: Let

$$
\begin{aligned}
S_{t}= & \left\{(a, b): 0 \leq a, b \leq 2^{n}-2,\right. \\
& \left.a+b \equiv t\left(\bmod 2^{n}-1\right), w(a)+w(b)<n\right\},
\end{aligned}
$$

where $1 \leq t \leq 2^{n}-2$. Then the cardinality $\left|S_{t}\right| \leq 2^{n-1}$.

Based on this conjecture, Tu and Deng [1] constructed two classes of Boolean functions with many good cryptographic properties. In this paper we always use the following bijection, where $X_{n}$ is the set of binary strings of length $n$ except the string consisting of $n$ copies of 1.

$$
\begin{aligned}
& Z_{2^{n-1}} \rightarrow X_{n} \\
& \sum_{i \leq n-1} x_{i} 2^{i} \mapsto x_{0} x_{1} \cdots x_{n-1}
\end{aligned}
$$

We use $|t|$ to denote the length of a binary string $t=t_{0} t_{1} \cdots t_{n-1}$. Let $-t=\left(1-t_{0}\right)\left(1-t_{1}\right) \cdots\left(1-t_{n-1}\right)$. And we use the following notation $1^{k} 0^{m}:=11 \cdots 100 \cdots 0$, where there are $k$ consecutive 1 and $m$ consecutive 0 in the string.

In [1] Tu and Deng construct an algorithm which they used it to show that the conjecture above is true when $n \leq 29$. Cusick, Li and Stanica [2] show that Conjecture
1 is true when $w(t) \leq 2$ or $w(t) \geq|t|-4$. In this paper, we will consider the following conjecture, which is equivalent to Conjecture 1.

Conjecture 2: Suppose that $1 \leq t \leq 2^{n}-2, n \geq 2$.

Let

$$
\begin{aligned}
S(t)= & \left\{a: 0 \leq a \leq 2^{n}-2, w(x) \geq w(a)+1,\right. \\
& \left.t+a \equiv x\left(\bmod 2^{n}-1\right), w(a)+w(b)<n\right\} .
\end{aligned}
$$

then $\left|S_{t}\right| \leq 2^{n-1}$.

The following lemma is easy so we omit the proof.

Lemma 1.1 Let $t=t_{0} t_{1} \cdots t_{n-1}$. Then following statements are true:

1) $|S(t)|=\left|S\left(t_{i} t_{i+1} \cdots t_{n-1} t_{0} t_{1} \cdots t_{i-1}\right)\right|$;

2) $w(t)+w(-t)=n$;

3) The map $\varphi: S_{t} \rightarrow S(-t), \varphi((a, b))=a$ is bijective. Hence $\left|S_{t}\right|=|S(-t)|$.

So the authors in [3] actually showed that Conjecture 2 is true when

$$
w(t) \geq|t|-2 \text { or } w(t) \leq 4 .
$$

According to Lemma 1.1. Deng and Yuan [4] show that Conjecture 2 is true if $w(t) \leq 6$.

The outline of this paper is as follows. In Section 2 we introduce some notations. In Section 3, we consider what happen if we change some digit 1 into 0 in the strings. We get a recursive formula about $S(t)$ and prove a new case of the conjecture.

\section{A Partition of $X_{n}$}

The following lemma is about the relation between $w(t+a)$ and $w(t)+w(a)$, which is proved in [4]. 


\section{Lemma 2.1 Let}

$$
t=t_{0} t_{1} \cdots t_{n-1} \in X_{n}, \quad a=a_{0} a_{1} \cdots a_{n-1} \in X_{n} .
$$

Suppose that

$$
I=\left\{j: 0 \leq j \leq n-1, t_{j}=a_{j}\right\}=\left\{i_{1}, i_{2}, \cdots, i_{l}\right\},
$$

where $0 \leq i_{1}<i_{2}<\cdots<i_{1} \leq n-1$. Assume that $t+a \neq 0^{n}$. Then

$$
w(t+a)=w(t)+w(a)-\sum_{s \in I, t_{i_{s}}=1}\left(i_{s+1}-i_{s}\right)
$$

where we set $i_{l+1}=i_{1}+n$.

Let

$$
S(t)=\left\{a \in X_{n}: w(t)+w(a)-w(t+a)=i\right\}
$$

for any $t \in X_{n}$ and $s(t)=\frac{|S(t)|}{2^{|t|}}$.

Then

$$
S(t)=\bigcup_{i=0}^{w(t)-1} S_{i}(t) \text { and } X_{n}=\bigcup_{i=0}^{|t|-1} S_{i}(t)
$$

which are disjoin unions. We define a partition on $X_{n}$ according to Lemma 2.1.

Definition 2.1 Let $t=t_{0} t_{1} \cdots t_{n-1}$ be a binary string of length $n$. Suppose that

$$
w(t)=r \text {, and } t_{m_{1}}=t_{m_{2}}=\cdots=t_{m_{r}}=1,
$$

where $0 \leq m_{1}<\cdots<m_{r} \leq n-1$. Let $a=a_{0} a_{1} \cdots a_{n-1}$ be a binary string. Suppose that

$$
I_{a}=\left\{j: 0 \leq j \leq n-1, t_{j}=a_{j}\right\}=\left\{i_{1}, i_{2}, \cdots, i_{l}\right\},
$$

where $0 \leq i_{1}<i_{2}<\cdots<i_{l} \leq n-1$. We set $\left(x_{1}, x_{2}, \cdots, x_{r}\right)^{t}$ to be the subset of $X_{n}$ such that $a \in\left(x_{1}, x_{2}, \cdots, x_{r}\right)^{t}$ if and only if the following two conditions hold

i) $x_{j}=i_{s+1}-i_{s}$, if $m_{j}=i_{s} \in I_{a}$;

ii) $x_{j}=0$, if $m_{j} \notin I_{a}$.

And we will use that notation $a^{t}:=\left(x_{1}, x_{2}, \ldots, x_{r}\right)^{t}$ if $a \in\left(x_{1}, x_{2}, \cdots, x_{r}\right)^{t}$.

Definition 2.2 Let $t=t_{0} t_{1} \cdots t_{n-1}$ and $a=a_{0} a_{1} \cdots a_{n-1}$ be two given binary strings. For any $0 \leq m \leq n-1$, we set $a_{m}^{t}=i$, if $b_{m}=i$ for each $b=b_{0} b_{1} \cdots b_{n-1} \in a^{t}, i=0,1$. We say that $a_{m}^{t}$ is free if there are two strings $b^{\prime}$ and $b^{\prime \prime}$ in $a^{t}$ such that $b_{m}^{\prime}=0$ and $b_{m}^{\prime \prime}=1$.

From Definition 2.1 and Lemma 2.1 we see that

$$
a^{t}=\left(x_{i}\right)^{t} \subseteq S_{\sum_{i=1}^{w(t)} x_{i}}(t) \text { and }\left|a^{t}\right|=2^{k},
$$

where $k$ is the number of indices such that $a_{i}^{t}$ is free.

\section{Example 2.1 Let}

$$
t=1110010010, a=1100101000, \quad b=1110010110,
$$

and

$$
C=1000110100 \text {. }
$$

Then

$$
t_{0}=t_{1}=t_{2}=t_{5}=t_{8}=1
$$

and

$$
\begin{aligned}
I_{a} & =\{0,1,3,5,7,9\}, \\
I_{b} & =\{0,1,2,3,4,5,6,8,9\}, \\
I_{c} & =\{0,3,5,6,9\} .
\end{aligned}
$$

So

$$
a \in(1,2,0,0,0)^{t}, \quad b \in(1,1,1,1,1)^{t} \text { and } c \in(3,0,0,1,0)^{t} \text {. }
$$

Moreover, by Definition $2.2 a^{\prime} \in a^{t}$ if and only if $a^{\prime}=1100 * 0 * * 0 *, *=0$ or 1 . That is, $a_{i}^{t}$ is free for $i=4,6,7,9$. We also have $b^{\prime} \in b^{t}$ if and only if

$$
b^{\prime}=1110 * 10 * 10, \quad c^{\prime} \in c^{t}
$$

if and only if

$$
c^{\prime}=1000 * 10 * 0 *, *=0 \text { or } 1 .
$$

\section{Main Results}

If $t=1^{r_{1}} 0^{s_{1}} 1^{r_{2}} 0^{s_{2}} \cdots 1^{r_{n}} 0^{s_{n}}$ with each $r_{i} \geq 1$, then we say that the block of $t$ is $n$. Jean-P. Flori and H. Randriam [5] give some asymptotic results when each $s_{i} \geq w(t)-1$. In particular, they show that Conjecture 2 is true if the block of $t$ is smaller than 3 or each $r_{i}$ is sufficient large for a fixed length of block. We give a recursive formula to show that we can restrict our attention to the case each $r_{i}$ is smaller than the block of $t$ in this situation. They also conjectured that

$$
\left|S\left(1^{r_{1}} 0^{s_{1}} \cdots 1^{r_{n}-1} 0^{s_{n}+1}\right)\right|>\left|S\left(1^{r_{1}} 0^{s_{1}} \cdots 1^{r_{n}} 0^{s_{n}}\right)\right| \text { if } r_{n}>3 .
$$

Lemma 3.1 Let

$$
t=10^{s_{1}} 10^{s_{2}} \cdots 10^{s_{r}}
$$

and

$$
T=10^{s_{1}} 10^{s_{2}} \cdots 10^{s_{r-2}} 10^{s_{r-1}+s_{r}+1}
$$

with

$$
s_{r} \geq r-1 \text { and }|t|=|T|=n \text {. }
$$

Let

$$
\begin{gathered}
m_{i}=\sum_{j=i}^{r-1}\left(s_{j}+1\right) \text { for } 1 \leq i \leq r-1, \\
\chi(T)=\left\{a \in X_{n}: a_{m_{1}}^{T} \text { is free }\right\}
\end{gathered}
$$

and

$$
\chi_{j}(T)=S_{j}(T) \cap \chi(T) .
$$

Then

$$
|S(T)|-|S(t)|=2^{-r}\left(\sum_{j=0}^{r-2} 2^{j}\left|\chi_{j}(T)\right|-2^{r-1}\left|\chi_{r-1}(T)\right|\right)
$$

Proof. Note that for any $\left(x_{1}, x_{2}, \cdots, x_{r}\right)^{t}$, if $x_{i} \geq m_{i}+1$, 
then $x_{j}=0$ for each $j>i$, moreover in this case we have

$$
\left|\left(x_{i}\right)^{t}\right|=\left|\left(x_{1}, \cdots, x_{i-1}, m_{i}, 0, \cdots, 0, x_{i}-m_{i}\right)^{t}\right| .
$$

Let

$$
\begin{aligned}
& I_{1}=\sum_{\sum x_{i} \leq r-1, x_{i}<m_{i}}\left|\left(x_{1}, x_{2}, \cdots, x_{r}\right)^{t}\right|, \\
& I_{2}=\sum_{\sum x_{i} \leq r-1, \text { one } x_{i} \leq m_{i}+1}\left|\left(x_{1}, x_{2}, \cdots, x_{r}\right)^{t}\right|,
\end{aligned}
$$

then $|S(t)|=I_{1}+2 I_{2}$. Similarly we write

$$
|S(T)|=J_{1}+J_{2} \text {, }
$$

where

$$
\begin{aligned}
& J_{1}=\sum_{\sum x_{i} \leq r-2, x_{i}<m_{i}}\left|\left(x_{1}, x_{2}, \cdots, x_{r-1}\right)^{T}\right|, \\
& J_{2}=\sum_{\sum x_{i} \leq r-2, \text { one } x_{i} \geq m_{i}}\left|\left(x_{1}, x_{2}, \cdots, x_{r-1}\right)^{T}\right| .
\end{aligned}
$$

We observe that if $a^{T}=\left(x_{1}, x_{2}, \cdots, x_{r-1}\right)^{T}$, then $a \in \chi$ if and only if each $x_{i}<m_{i}$. Now if $x_{i} \geq m_{i}+1$, by comparing the number of free indices we have

$$
\left|\left(x_{1}, \cdots, x_{i}, 0, \cdots, 0\right)^{t}\right|=\frac{1}{2}\left|\left(x_{1}, \cdots, x_{i}, 0, \cdots, 0\right)^{T}\right| .
$$

Hence, $2 I_{2}=J_{2}$. If each $x_{i}<m_{i}$, then

$$
\left|\left(x_{1}, \cdots, x_{r}\right)^{t}\right|=2^{-x_{r}-1}\left|\left(x_{1}, \cdots, x_{r-1}\right)^{T}\right| \text {. }
$$

Suppose that $\sum_{i=1}^{r-1} x_{i}=j \leq r-1$. Then

$$
\begin{aligned}
\sum_{x_{r}=0}^{r-1-j}\left|\left(x_{1}, \cdots, x_{r}\right)^{t}\right| & =\sum_{x_{r}=0}^{r-1-j} 2^{-x_{r}}\left|\left(x_{1}, \cdots, x_{r-1}, 0\right)^{t}\right| \\
& =2\left(1-2^{-r+j}\right)\left|\left(x_{1}, \cdots, x_{r-1}, 0\right)^{t}\right| .
\end{aligned}
$$

So

$$
\begin{aligned}
I_{1} & =\sum_{j=0}^{r-1} \sum_{\sum x_{i} \leq r-1, x_{i}<m_{i}} 2\left(1-2^{-r+j}\right)\left|\left(x_{1}, \cdots, x_{r-1}, 0\right)^{t}\right| \\
& =\sum_{j=0}^{r-1} \sum_{\sum x_{i} \leq r-1, x_{i}<m_{i}}\left(1-2^{-r+j}\right)\left|\left(x_{1}, \cdots, x_{r-1}\right)^{T}\right| .
\end{aligned}
$$

Therefore

$$
\begin{aligned}
& |S(T)|-|S(t)|=J_{1}-I_{1} \\
& \quad \sum_{\sum x_{i} \leq r-2, x_{i}<m_{i}}\left|\left(x_{1}, x_{2}, \cdots, x_{r-1}\right)^{T}\right| \\
& -\sum_{j=0}^{r-1} \sum_{\sum x_{i} \leq r-1, x_{i}<m_{i}}\left(1-2^{-r+j}\right)\left|\left(x_{1}, \cdots, x_{r-1}\right)^{T}\right| \\
& =2^{-r}\left(\sum_{j=0}^{r-2} 2^{j}\left|\chi_{j}(T)\right|-2^{r-1}\left|\chi_{r-1}(T)\right|\right) .
\end{aligned}
$$

This finishes the proof.

Remark 3.1 Let $t=10^{s_{1}} 10^{s_{2}} \cdots 10^{s_{r}}$ with $s_{r} \geq r-1$.
It is clear that

$$
\left|S\left(t 0^{n}\right)\right|=2^{n}|S(t)|
$$

for any $n>0$. So we use the following notation $0^{\infty}$ means that there are sufficient consecutive 0 in the string. We set

$$
\chi_{j}(t)=\left\{a: a^{t}=\left(x_{i}\right)^{t}, x_{j}=0 \text { for } j>\sum_{i=1}^{n-1} r_{i}\right\}
$$

for $t=1^{r_{1}} 0^{\infty} 1^{r_{2}} 0^{\infty} \cdots 1^{r_{n}} 0^{\infty}$.

\section{Theorem 3.1 Let}

$$
\begin{aligned}
& t=1^{r_{1}} 0^{\infty} 1^{r_{2}} 0^{\infty} \cdots 1^{r_{n}-1} 0^{\infty}, \quad T=1^{r_{1}} 0^{\infty} 1^{r_{2}} 0^{\infty} \cdots 1^{r_{n}} 0^{\infty}, \\
& t^{\prime}=1^{r_{1}-1} 0^{\infty} 1^{r_{2}} 0^{\infty} \cdots 1^{r_{n}} 0^{\infty}, \quad T^{\prime}=1^{r_{1}-1} 0^{\infty} 1^{r_{2}} 0^{\infty} \cdots 1^{r_{n}+1} 0^{\infty}, \\
& t^{*}=10^{\infty} 1^{r_{2}} 0^{\infty} \cdots 1^{r_{n}-2} 0^{\infty}, \quad T^{*}=10^{\infty} 1^{r_{2}} 0^{\infty} \cdots 1^{r_{n}-1} 0^{\infty},
\end{aligned}
$$

where each $r_{i} \geq 1$ and $r_{1} \geq 2, r_{n} \geq 2$. Then

$$
s(t)-s(T)=s\left(t^{\prime}\right)-s\left(T^{\prime}\right)+2^{-2}\left(s\left(t^{*}\right)-s\left(T^{*}\right)\right) .
$$

Proof. Suppose that those strings have the same length and $w(T)=\sum_{i=1}^{n} r_{i}=r$. By Lemma 3.1

$$
\begin{aligned}
& |S(t)|-|S(T)|=2^{-r}\left(\sum_{j=0}^{r-2} 2^{j}\left|\chi_{j}(t)\right|-2^{r-1}\left|\chi_{r-1}(t)\right|\right) \\
& \left|S\left(t^{\prime}\right)\right|-\left|S\left(T^{\prime}\right)\right|=2^{-r}\left(\sum_{j=0}^{r-2} 2^{j}\left|\chi_{j}\left(t^{\prime}\right)\right|-2^{r-1}\left|\chi_{r-1}\left(t^{\prime}\right)\right|\right) \\
& \left|S\left(t^{*}\right)\right|-\left|S\left(T^{*}\right)\right| \\
& =2^{-r+r_{1}}\left(\sum_{j=0}^{r-r_{1}-2} 2^{j}\left|\chi_{j}\left(t^{*}\right)\right|-2^{r-r_{1}-1}\left|\chi_{r-r_{1}-1}\left(t^{*}\right)\right|\right) .
\end{aligned}
$$

Let

$$
a^{t}=\left(x_{11}, \cdots, x_{1 r_{1}}, \cdots, x_{n-1,1}, \cdots, x_{n-1, r_{n-1}}, 0, \cdots, 0\right)^{t} .
$$

If $x_{11}>0$, by comparing the number of free indices we have

$$
\left|a^{t}\right|=\left|\left(r_{1}-1,0, \cdots, y, x_{21}, \cdots, x_{n-1, r_{n-1}}, 0, \cdots, 0\right)\right|^{t},
$$

where $y=\sum_{i=1}^{r_{1}} x_{1 i}-r_{1}+1$. We set

$$
\kappa_{j}(t)=\left\{a \in \chi_{j}(t): a^{t}=\left(0, y_{2}, \cdots, y_{r-1}\right)^{t}\right\}
$$

and

$$
\lambda_{j}(t)=\left\{a \in \chi_{j}(t): a^{t}=\left(r_{1}-1, y_{2}, \cdots, y_{r-1}\right)^{t}\right\} .
$$

Then

$$
\begin{aligned}
|S(t)|-|S(T)|= & 2^{-r}\left(\sum_{j=0}^{r-2} 2^{j}\left|\kappa_{j}(t)\right|-2^{r-1}\left|\kappa_{r-1}(t)\right|\right) \\
& +2^{-r+r_{1}-1}\left(\sum_{j=0}^{r-2} 2^{j}\left|\lambda_{j}(t)\right|-2^{r-1}\left|\lambda_{r-1}(t)\right|\right)
\end{aligned}
$$

Let

$$
I_{1}=2^{-r}\left(\sum_{j=0}^{r-2} 2^{j}\left|\kappa_{j}(t)\right|-2^{r-1}\left|\kappa_{r-1}(t)\right|\right),
$$


and

$$
I_{2}=2^{-r+r_{1}-1}\left(\sum_{j=0}^{r-2} 2^{j}\left|\lambda_{j}(t)\right|-2^{r-1}\left|\lambda_{r-1}(t)\right|\right) .
$$

Now consider the following mapping $\varphi$ and $\psi$. If $a \in \kappa_{j}(t)$ and $a^{t}=\left(0, y_{2}, \cdots, y_{r-1}\right)^{t}$, then

$$
\varphi\left(a^{t}\right)=\left(y_{2}, \cdots, y_{r-1}, 0\right)^{t^{\prime}}
$$

Then

$$
\left|a^{t}\right|=\left|\varphi\left(a^{t}\right)\right|
$$

So

$$
\left|\kappa_{j}(t)\right|=\left|\chi_{j}\left(t^{\prime}\right)\right|
$$

If

$$
a \in \lambda_{j}(t) \text { and } a^{t}=\left(r_{1}-1, y_{2}, \cdots, y_{r-1}\right)^{t} \text {, }
$$

then

$$
\psi\left(a^{t}\right)=\left(y_{r_{1}}-1, y_{r_{1}+1}, \cdots, y_{r-1}\right)^{t^{*}} .
$$

It is easy to see that

$$
\psi\left(a^{t}\right) \subseteq \chi_{j-r_{1}}\left(t^{*}\right) \text { and }\left|a^{t}\right|=2^{-r_{1}-1}\left|\psi\left(a^{t}\right)\right| .
$$

By the discussion above we obtain

$$
I_{1}=\left|S\left(t^{\prime}\right)\right|-\left|S\left(T^{\prime}\right)\right| \text {, and } I_{2}=2^{-2}\left(\left|S\left(t^{*}\right)\right|-\left|S\left(T^{*}\right)\right|\right) \text {. }
$$

This finishes the proof.

Corollary 3.1 With the same notations in Theorem 3.1. Suppose that

$$
r_{n} \geq \#\left\{i: 1 \leq i \leq n, r_{i} \geq 2\right\}
$$

then

$$
s(t)>s(T) .
$$

Proof. We proof the statement by induction on

$$
l=r_{n} \geq \#\left\{i: 1 \leq i \leq n, r_{i} \geq 2\right\} .
$$

The case $l=1$ implies that

$$
r_{1}=r_{2}=\cdots=r_{n-1}=1 \text {. }
$$

This was proved in [4]. Without loss of generality we can assume that $r_{1}>1$ and $r_{n}>l$. By induction

$$
s\left(t^{*}\right)>s\left(T^{*}\right)
$$

by Theorem 3.1

$$
s(t)-s(T)>s\left(t^{\prime}\right)-s\left(T^{\prime}\right) .
$$

The proof is completed by induction on $\sum_{i=1}^{n-1} r_{i}$.

Corollary 3.2 Let $t=1^{r_{1}} 0^{\infty} 1^{r_{2}} 0^{\infty} 1^{r_{3}} 0^{\infty}$. Then

$$
\mathrm{s}(t) \leq \frac{1}{2} \text {. }
$$

Proof. By Corollary 3.1 it suffice to show that case when each $r_{i}<3$. So we have $w(t) \leq 6$, which is proved in [4].

\section{REFERENCES}

[1] Z. Tu and Y. Deng, "A Conjecture on Binary String and Its Application on Constructing Boolean Functions of Optimal Algebraic Immunity,” Designs, Codes and Cryptography, Vol. 60, No. 1, 2010, pp. 1-14. doi:10.1007/s10623-010-9413-9

[2] G. Cohen and J.-P. Flori, "On a Generalized Combinatorial Conjecture Involving Addition Mod $2^{k}-1$, IACR Cryptology ePrint Archive, Vol. 400, 2011, in press.

[3] T. W. Cusick, Y. Li and P. Stanica, “On a Combinatorial Conjecture,” Integers, Vol. 11, No. 2, 2011, pp. 185-203. doi:10.1515/integ.2011.017

[4] G. Deng and P. Yuan, "On a Combinatorial Conjecture of Tu and Deng,” Integers, Vol. 12, No. A48, 2012.

[5] J.-P. Flori and H. Randriam, "On the Number of Carries Occuring in an Addition Mod $2^{k}-1$," Integers, Vol. 12, No. A10, 2012. 